\title{
Biosynthesis of Silver Nanoparticles by Marine Invertebrate (Polychaete) and Assessment of Its Efficacy against Human Pathogens
}

\author{
Reena Singh, Sunil Kumar Sahu, and Muthusamy Thangaraj \\ Centre of Advanced Study in Marine Biology, Faculty of Marine Sciences, Annamalai University, \\ Parangipettai, Tamil Nadu 608 502, India
}

Correspondence should be addressed to Muthusamy Thangaraj; coralholders@gmail.com

Received 11 May 2014; Revised 24 July 2014; Accepted 29 July 2014; Published 21 August 2014

Academic Editor: Xiangwu Zhang

Copyright (C) 2014 Reena Singh et al. This is an open access article distributed under the Creative Commons Attribution License, which permits unrestricted use, distribution, and reproduction in any medium, provided the original work is properly cited.

\begin{abstract}
Synthesis of metallic nanoparticles by chemical and physical method makes the process often cumbersome due the usage of toxic and expensive chemicals. The present study reports the biosynthesis of silver nanoparticles using marine invertebrate (polychaete) extract at room temperature. The ultraviolet-visible (UV-Vis) spectroscopy revealed the formation of silver nanoparticles (AgNPs) by exhibiting the typical surface plasmon absorption maximum at $418-420 \mathrm{~nm}$. Structure and composition of AgNPs were analyzed by atomic force microscopy (AFM). Average particle size of AgNPs ranged from 40 to $90 \mathrm{~nm}$, confirmed by scanning electron microscopy (SEM) analysis. The energy-dispersive X-ray spectroscopy (EDX) of the nanoparticles dispersion confirmed the presence of elemental silver signal, whereas X-ray diffraction (XRD) substantiated the crystalline nature of synthesized nanoparticle. Fourier transform infrared spectroscopy (FTIR) spectral analysis showed the presence of amides phenols, ethers, and fatty acids as major biomolecules responsible for the reduction of silver ions. The possible mechanism responsible for the synthesis of AgNPs by these biomolecules was also illustrated by chemical reactions. The synthesized AgNPs showed comparatively good antibacterial activity against the tested human pathogens. This study advocates that not only plants and microbes but also marine invertebrates do have potential for synthesizing nanoparticles by a cost-effective and eco-friendly approach.
\end{abstract}

\section{Introduction}

Colloidal particles are receiving augmented attention as an important starting point for the fabrication of micro- and nanostructures due to their attractive physical and chemical properties which differ considerably from the bulk phase [1]. The integration of nanomaterials with biology has led to the development of diagnostic devices, various analytical tools, therapeutic applications, and drug delivery vehicles [2]. Silver nanoparticles (AgNPs) have high reactivity due to the large surface to volume ratio and play a crucial role in inhibiting bacterial growth in aqueous and solid media. For instance, AgNPs have been reported to possess antitumour [3], antibacterial [4], antifungal [5], and antiviral activity [6]. The antimicrobial activity of AgNPs is influenced by the dimensions of the particles; usually the smaller the particles, the greater the antimicrobial effect [7]. The production of nanoparticles with desired shape and size can be obtained from simple bacteria to highly complex eukaryotes in the reaction mixture $[4,8]$.

However, with the development of new chemical and physical methods for the synthesis of nanoparticles, the concern for environmental contaminations is also increasing. Living organisms have enormous potential for the production of nanoparticles. The ability of plants and microbes to produce nanoparticles has featured exciting loom towards the escalation of natural nanofactories. This phenomenon has brought an insight among researchers for the development of molecules by using an elegant and ingenious method which is precise and efficient [9]. The synthesis of nanoparticles by using biological systems is advantageous over chemical and physical methods as it is cost effective and environmentfriendly which does not require high pressure, energy, temperature, or toxic chemicals [10]. 
In recent years, many bioactive compounds and nanoparticles have been synthesized from various terrestrial and marine organisms [11]. However, such attempts are curbed mainly to plants and microbes [12]. There is dearth of information available regarding the synthesis of nanoparticles from marine invertebrates. Polychaetes, the major taxonomic group in estuarine and marine ecosystems, occupy a large infaunal habitat and play an important role in bioturbation, transfer of organic materials and nutrients from the overlying water column to the sediment and vice versa $[13,14]$. Polychaetes are valued by the aquaculture industry as an excellent source of polyunsaturated fatty acids (PUFAs), and they have the potential to supplement fish oil as sources of essential lipid components of feeds [15] and hence they are also entitled as Omega worms [16]. Therefore, in the present study an attempt was made to address the following questions. (a) Is polychaete extract capable of synthesizing AgNPs? (b) If yes, then what is the size of the nanoparticle? What are its further characterizations by analytical tools? (c) How efficient are the nanoparticles synthesized from polychaete against human pathogen?

\section{Materials and Methods}

2.1. Chemicals. All analytical reagents and bacteriological media were purchased from Hi-Media Laboratories, Mumbai, India. All media and solutions were prepared in doubledistilled Milli-Q water. All the experiments were performed in triplicate.

2.2. Sample Collection. Polychaetes were collected during the low tide from sediments of Uppanar estuary (latitude $11^{\circ} 40^{\prime} 20.29 \mathrm{~N}$; longitude $\left.79^{\circ} 45^{\prime} 15.79 \mathrm{E}\right)$ Cuddalore, Tamil $\mathrm{Nadu}$, and washed thoroughly with distilled water to remove the unsolicited dirt particles.

2.3. Preparation of Polychaete Extract. The polychaete sample $(\sim 10 \mathrm{~g})$ was finely pulverized using mortar and pestle. The extract was made up to $100 \mathrm{~mL}$ using double-distilled Milli$\mathrm{Q}$ water. Then the extract was filtered through Whatman number 1 filter paper to separate the tissue rubbles and obtain a pure extract.

2.4. Synthesis of Silver Nanoparticles. The polychaete filtrate was used as reducing agent and stabilizer for the synthesis of AgNPs. $10 \mathrm{~mL}$ of the filtrate was mixed with $90 \mathrm{~mL}$ of $1 \mathrm{mM}$ silver nitrate solution in a $250 \mathrm{~mL}$ Erlenmeyer flask and agitated at room temperature in dark. A flask containing $10 \mathrm{~mL}$ Milli-Q and $90 \mathrm{~mL} 1 \mathrm{mM}$ silver nitrate solution was taken as control. The change in colour was visually monitored till the appearance of typical dark brown colour.

\subsection{Characterization of Nanoparticles}

2.5.1. Spectroscopic Validation. After 24 hours of incubation, one $\mathrm{mL}$ of sample was withdrawn every six hours and the optical density (OD) was taken at a broad range of wavelengths from 300 to $700 \mathrm{~nm}$ using a UV-visible spectrophotometer (UV 2450, Shimadzu) and the graph was plotted based on the OD readings by automated software UV Probe.

2.5.2. Atomic Force Microscopy. In order to further characterize the size and dispersion of the silver nanoparticles, the colloidal solution was dried as a thin layer on mica-based glass slide $(1 \mathrm{~mm} \times 1 \mathrm{~mm} \times 1 \mathrm{~mm})$ followed by visualization under the atomic force microscope (AFM), model N9410A series 5500, Agilent.

2.5.3. X-Ray Diffraction Pattern. The X-ray diffraction (XRD) measurement of silver nanoparticles was carried out using $\mathrm{Cu}-\mathrm{K} \alpha$ radiation source in a wide range of Bragg angles $2 \theta$ at a scanning rate of $0.388 / \mathrm{min}$ in powder diffractometer (PANalyticalX' per PRO model X-ray diffractometer), at the voltage of $50 \mathrm{kV}$ and a current of $30 \mathrm{~mA}$.

2.5.4. Scanning Electron Microscopy (SEM) and EnergyDispersive X-Ray (EDX) Analysis. The sample was kept on copper grid stained with uranyl acetate and lead citrate and was observed under JEOL-JSM-5610LV scanning electron microscope (SEM). Energy-dispersive X-ray spectroscopy (EDS) analysis was also carried out for the detection of elemental silver by using INCA EDS.

2.5.5. Fourier Transform Infrared Spectroscopy (FTIR) Measurements. The polychaete sample before and after the synthesis of AgNPs was freeze-dried and pelleted with potassium bromide $(\mathrm{KBr})$ in the ratio of $1: 10$ and subjected for FTIR spectroscopic measurement (Nicolet IS5, Thermo Scientific). The wavenumber ranged from 450 to $2500 \mathrm{~cm}^{-1}$ with the resolution of $4 \mathrm{~cm}^{-1}$ and was analyzed by subtracting the spectrum of pure $\mathrm{KBr}$.

2.5.6. Assessment of Antibacterial Activity. Antibacterial activity of the synthesized AgNPs was determined by using the Kirby-Bauer disc diffusion method [17] against five human pathogens, namely, Staphylococcus aureus, Escherichia coli, Pseudomonas aeruginosa, Vibrio parahaemolyticus, and Salmonella typhi. All the pathogenic bacterial strains were obtained from Raja Muthiah Medical College, Annamalai University, Tamil Nadu, India. Stock cultures were maintained at $4^{\circ} \mathrm{C}$ on agar slants of nutrient media. Prior to the experiment, pure cultures were subcultured in Muller Hinton broth and incubated overnight at $37^{\circ} \mathrm{C}$. The inoculums suspensions were swabbed uniformly in different Petri plates. Filter paper discs saturated with AgNPs were placed aseptically in the plates with the help of sterile forceps and incubated at $37^{\circ} \mathrm{C}$. Tetracycline and distilled water were taken as positive and negative control, respectively. After 24 hours of incubation, the zone of inhibition was observed and measured.

\section{Results}

The successful synthesis of AgNPs by polychaete extract was evident by the formation of dark brown colour. The control 


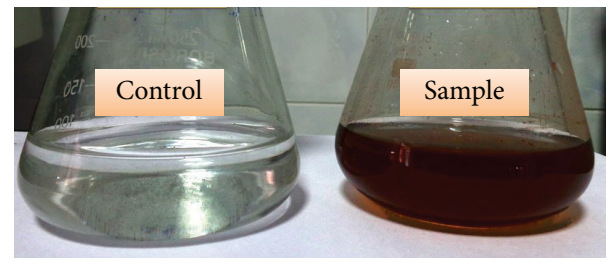

FIGURE 1: Visual observation of colour change in control and synthesized silver nanoparticles.

$\left(1 \mathrm{mM} \mathrm{AgNO}_{3}\right)$ solution does not exhibit any color change (Figure 1). The gradual change in colour was observed immediately after two hours of incubation. However, fully dark brown colour appeared only after 72 hours. But the handling and processing of the extract were less stringent compared to chemical and physical methods. The intermittent UVVis spectral observation of the synthesized nanoparticle showed no significant shift in the absorbance intensity as well as absorption maxima indicating the uniform particle size throughout the experiment.

3.1. UV-Vis Spectra Analysis. The UV-visible spectrophotometric analysis of colloidal reaction mixture of the synthesized AgNPs showed peak at $418-420 \mathrm{~nm}$ in the spectrum. The stability of the nanoparticle was also verified. No precipitation was observed in the reaction mixture for the period of six months. The evidence of surface plasmon resonance phenomenon (SPR) is shown in Figure 2.

3.2. Atomic Force Microscopic Analysis. The atomic force microscopy (AFM) results displayed the surface morphology of the polydispersed AgNPs. The particle size of the AgNPs ranged from 40 to $90 \mathrm{~nm}$. The topographical image of AgNPs in particular bright spots indicated that they are agglomerated and formed distinct nanoparticles mostly spherical in shape (Figures 3(a) and 3(b)).

3.3. SEM and EDS Analysis. Scanning electron microscopy provided further insight into the morphology and size details of the synthesized nanoparticles. The SEM image showed relatively spherical and triangular shaped nanoparticle with diameter ranging from 40 to $90 \mathrm{~nm}$ (Figure 4(a)). This further substantiated the result of AFM analysis. The SEM image also showed polydispersed nanoparticles on the surface. Energydispersive X-ray (EDX) analysis further confirmed the presence of elemental silver signals of the silver nanoparticles (Figure 4(b)).

3.4. X-Ray Diffraction Study. The XRD pattern of the synthesized nanoparticles corresponds to that of silver nanoparticles. The XRD pattern shows four intense peaks in the whole spectrum of $2 \theta$ values ranging from 30 to 80 . Comparative analysis of the XRD spectrum with the standard confirmed that the synthesized silver particles were in the form of nanocrystals, evident by the peaks at $2 \theta$ values of $38.25^{\circ}$, $46.37^{\circ}, 64.60^{\circ}$, and $77.62^{\circ}$, corresponding to $111,200,220$, and 311 planes for silver, respectively (Figure 5).

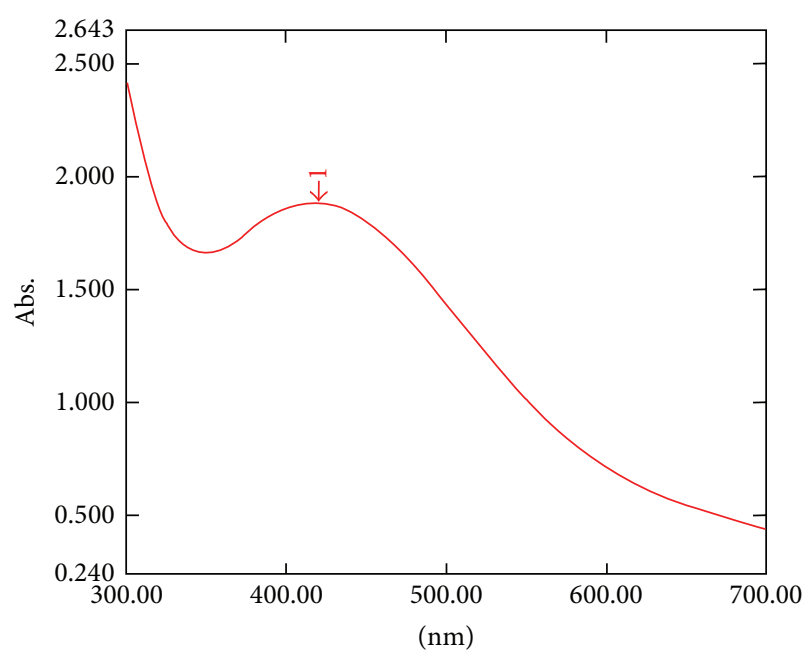

FIGURE 2: UV-Vis absorption spectra of silver nanoparticles synthesized from polychaete extract.

3.5. FTIR Analysis and Possible Mechanism for the Synthesis of AgNPs. To identify the possible biomolecules responsible for efficient stabilization of the silver nanoparticles, the polychaete extract and the synthesized nanoparticle were subjected for FTIR spectral studies. The comparative FTIR profile, before and after synthesis of AgNPs, is depicted in Figure 6. The spectra revealed the presence of prominent peaks at $3345,2922,1670,1384,1088$, and $1037 \mathrm{~cm}^{-1}$ corresponding to different functional groups, namely, $\mathrm{NH}$, $\mathrm{OH}, \mathrm{C} \equiv \mathrm{N}, \mathrm{N}-\mathrm{O}, \mathrm{C}=\mathrm{C}, \mathrm{C}-\mathrm{N}$, and $\mathrm{C}=\mathrm{CH}_{2}$, in synthesized nanoparticles, analogous to the well-known signatures in the infrared region of the electromagnetic spectrum (Figure 6). Before synthesis of AgNPs, mainly 3408, 2931, 1645, and 1033 were the prominent peaks conforming the presence of $\mathrm{C}-\mathrm{X}$, $\mathrm{C}-\mathrm{O}, \mathrm{C}-\mathrm{C}, \mathrm{C}=\mathrm{O}, \mathrm{C}-\mathrm{H}$, and $\mathrm{N}-\mathrm{H}$ groups. The possible chemical reaction which might be responsible for the synthesis of AgNPs is illustrated in Figure 7. The structures were used based on our previous findings using GC-MS analysis [14].

3.6. Antibacterial Activity. Antimicrobial effects of the synthesized silver nanoparticles were evaluated against five human pathogens (Figure 8). The maximum antibacterial activity was observed against Staphylococcus aureus which showed $13 \mathrm{~mm}$ zone of inhibition followed by Escherichia coli $(10 \mathrm{~mm})$, Pseudomonas aeruginosa $(10 \mathrm{~mm})$, and Salmonella typhi $(9 \mathrm{~mm})$. The lowest was noticed against Vibrio parahaemolyticus $(8 \mathrm{~mm})$. However, from Figure 7 it is quite evident that the positive control (tetracycline) also showed better zone of inhibition ranging from 12 to $14 \mathrm{~mm}$.

\section{Discussion}

Owing to the applicability of silver nanoparticles in wide sectors, its demand is increasing at an overwhelming rate which has resulted in increased production. Researchers are continuously developing newer methods for synthesis of highly monodispersed silver nanoparticles which are efficient 


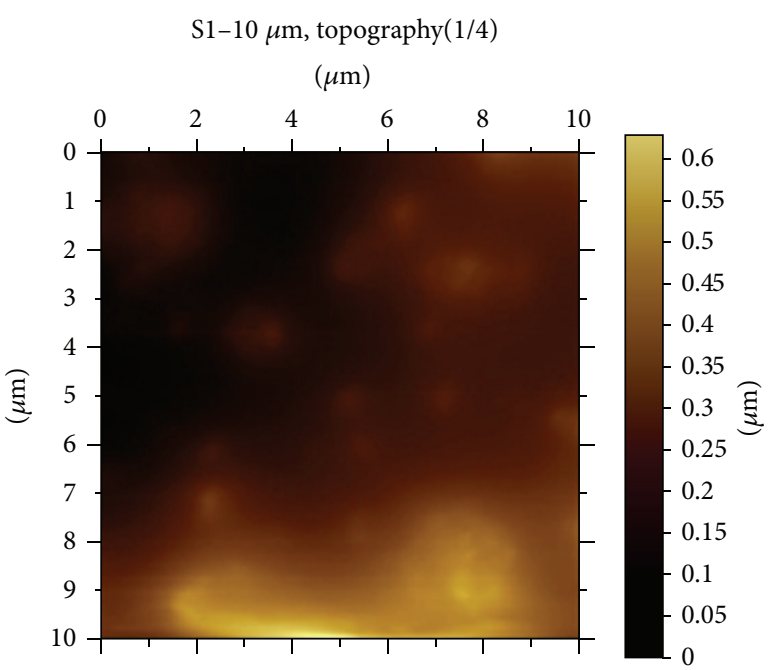

(a)

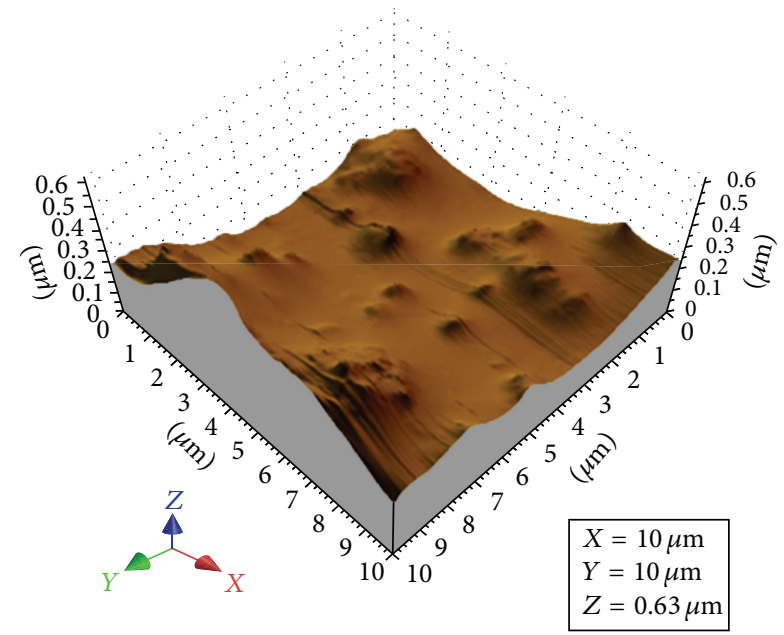

(b)

FIGURE 3: AFM topography of synthesized silver nanoparticles by the polychaete extract.

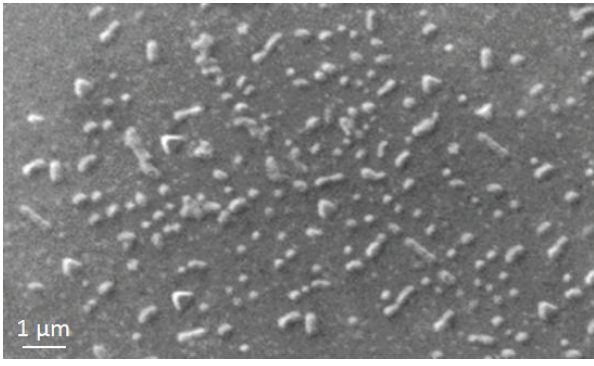

(a)

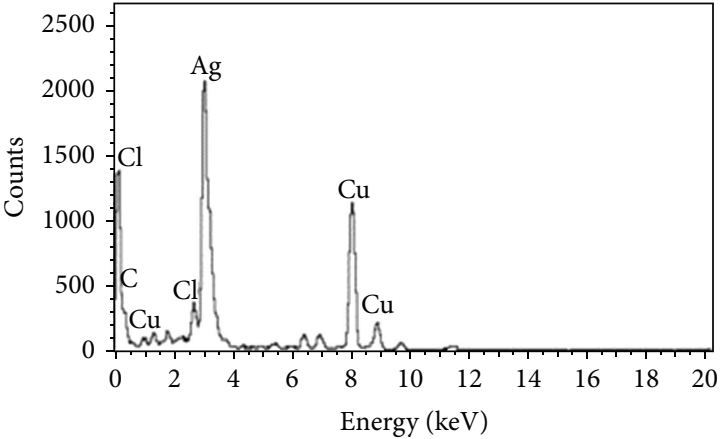

(b)

FIGURE 4: (a) SEM image and (b) energy-dispersive X-ray spectroscopy (EDS) analysis confirming the presence of elemental silver. The scale bar corresponds to $1 \mu \mathrm{m}$.

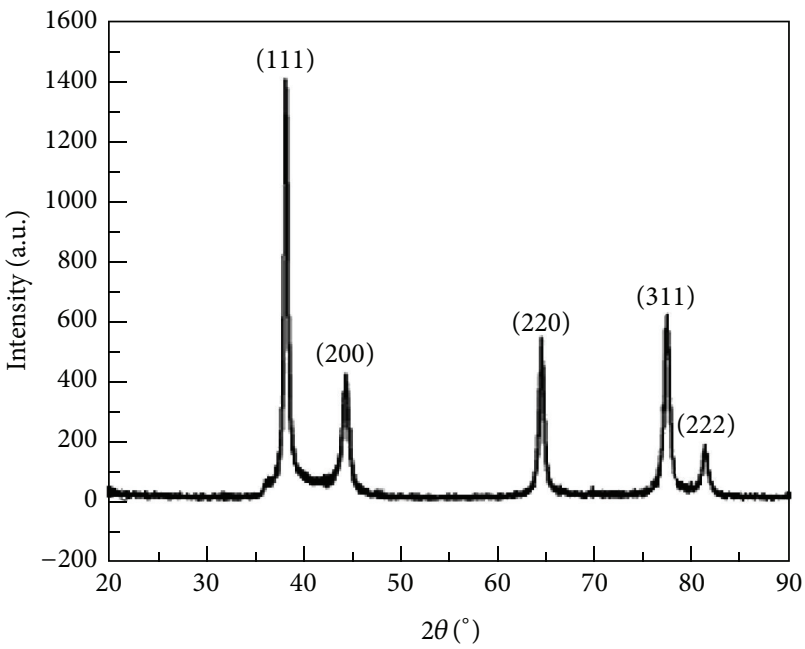

FIGURE 5: X-ray diffraction pattern of the silver nanoparticles. in terms of synthesis rate as well as energy usage. Biological methods have emerged as an alternative to the conventional methods for synthesis of nanoparticles. The appearance of dark brown is a clear indication of the formation of silver nanoparticles formed in the reaction mixture [18]. Marine source has recently been explored for the synthesis of gold nanoparticles from sponge, Acanthella elongata [1]. However, the present report is the first attempt to test the efficacy of marine polychaete for the synthesis of silver nanoparticle. Synthesis of inorganic nanoparticles by biological systems makes nanoparticles more biocompatible and environmentally benign [19] and cost effective [20].

Silver nanoparticles exhibit new optical properties, which are observed neither in molecules nor in bulk metals. The synthesized nanoparticle exhibited peak at $418-420 \mathrm{~nm}$ in the spectrum. This band appears due to the surface plasmonoscillation modes of conduction electrons which are coupled through the surface to external electromagnetic fields [21]. 


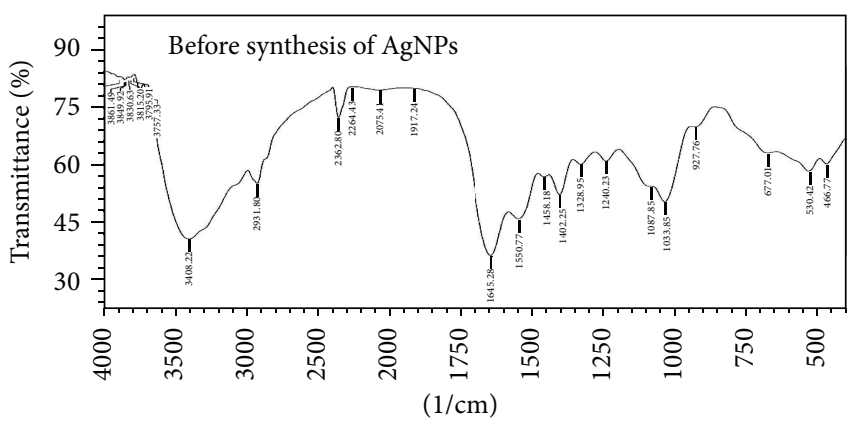

(a)

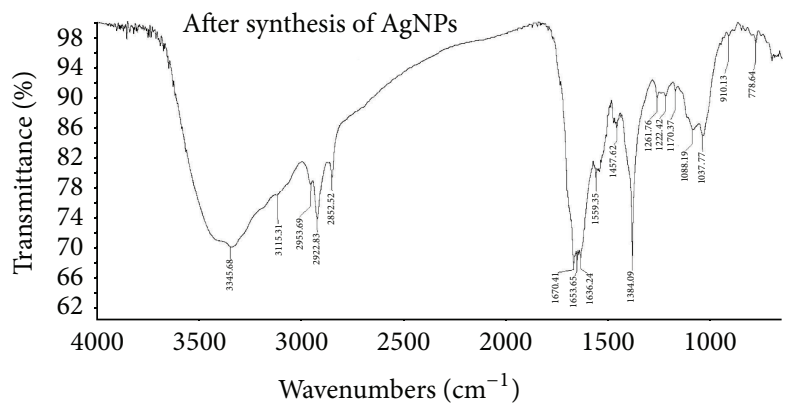

(b)

FIGURE 6: FTIR spectrum (transmittance mode) of the polychaete extract after synthesis of silver nanoparticles.

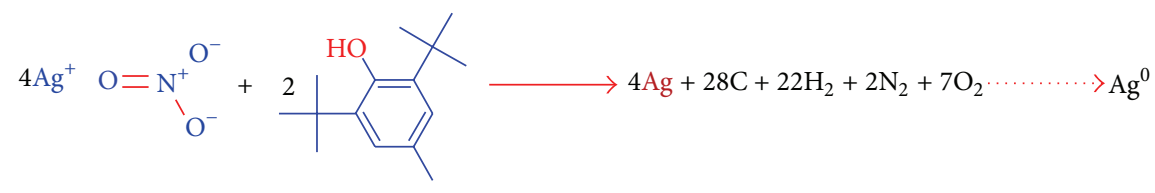

(a)

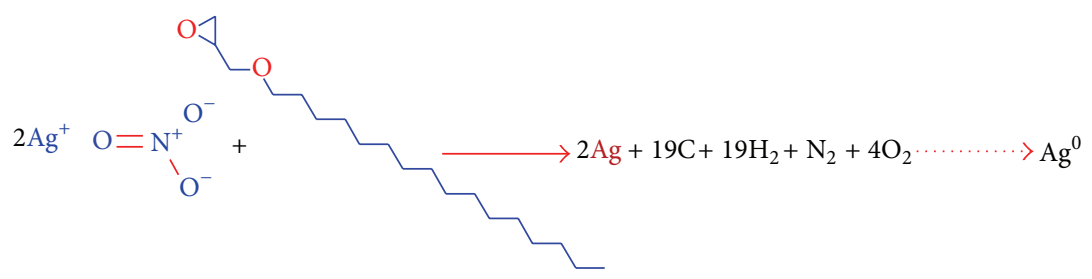

(b)

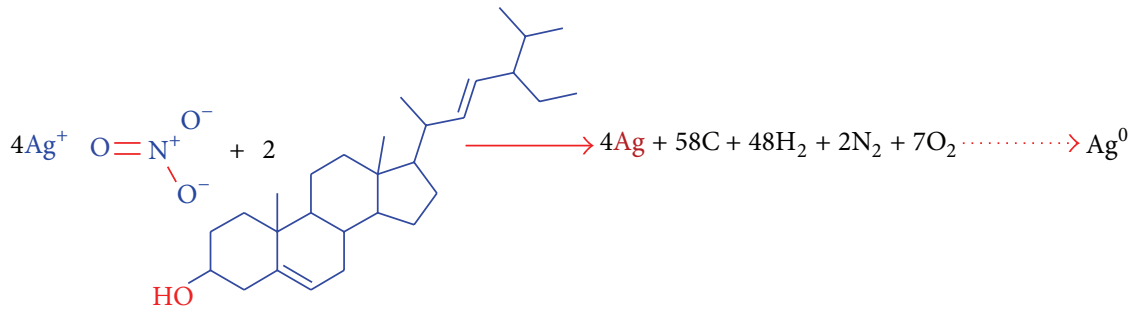

(c)

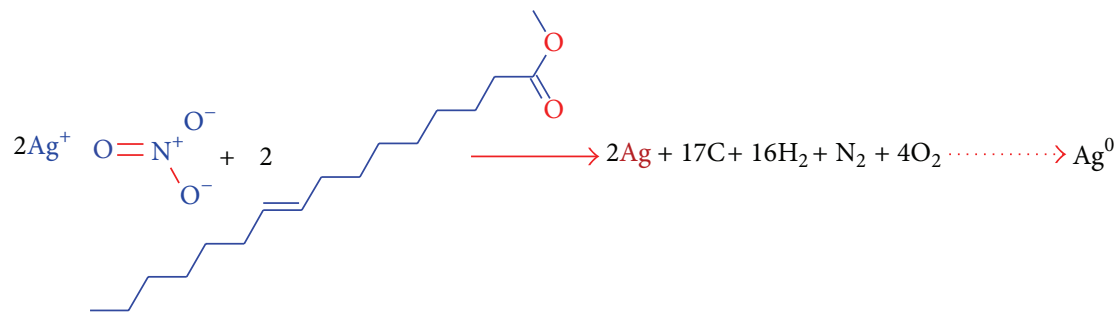

(d)

FIGURE 7: Possible mechanism of reaction for the synthesis of silver nanoparticles by the active molecules: (a) 2,4-di-tert-butylphenol, (b) glycidyl hexadecyl ether, (c) stigmasterol, and (d) 9-hexadecenoic acid.

Electrons are limited to specific vibration modes by the particle's size and shape. Therefore, metallic nanoparticles have characteristic optical absorption spectrums in the UVVis region [22]. Many plants and bacteria as well as fungal species have been used for silver nanoparticle synthesis [23].
But most of them have been reported to accumulate AgNPs intracellularly. Intracellular synthesis always takes longer reaction times and also demands subsequent extraction and recovery steps [24]. On the contrary, the present study reports the successful biosynthesis of AgNPs from marine 


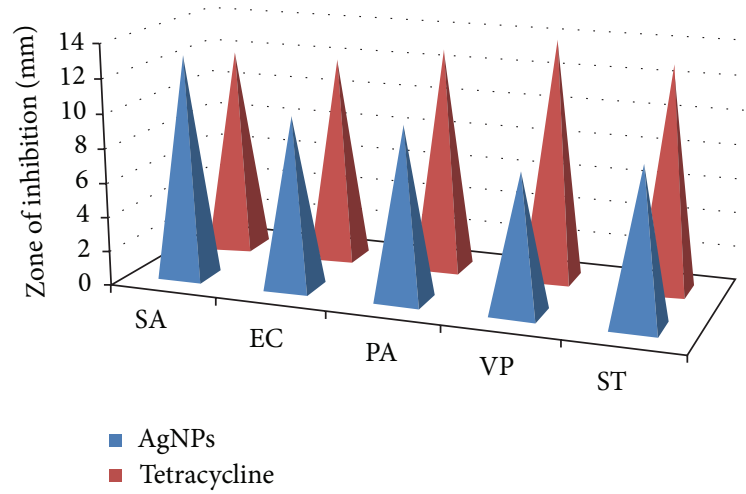

FIGURE 8: Antibacterial activity of silver nanoparticle against human pathogens. Blue cone represents silver nanoparticles (AgNPs), whereas positive control (tetracycline) is depicted by red cone. Here, SA stands for Staphylococcus aureus; EA: Escherichia coli; PA: Pseudomonas aeruginosa; VP: Vibrio parahaemolyticus; and SA: Salmonella typhi.

polychaetes with longer reaction time ( 72 hours) for complete synthesis. The exact reasons and mechanisms need to be explored in this particular species.

AFM analysis showed that the particle size of the AgNPs ranged from 40 to $90 \mathrm{~nm}$. The topographical image indicated agglomeration of the nanoparticle which could be attributed to the fact that AgNPs tend to form aggregates on the surface during deposition [25]. Scanning electron microscopy image showed spherical and triangular shaped polydispersed nanoparticles. The shape and size of the metal nanoparticles considerably change the optical and electronic properties [26]. The XRD patterns clearly revealed the crystalline nature of the biosynthesized silver nanoparticles. The peaks corresponding to the $2 \theta=38.25^{\circ}$ (111), $46.37^{\circ}$ (200), $64.60^{\circ}$ (220), and $77.62^{\circ}$ (311) of the sample respect Bragg's model of diffraction given by Joint Committee on Powder Diffraction Standards (JCPDS, file numbers 04-0783 and 84-0713). FTIR has become an important tool in understanding the involvement of functional groups in relation between metal particles and biomolecules. The amide group corresponds to the presence of enzymes which are further prerequisite for the reduction synthesis and stabilization of the metal ions [27]. The presence of other functional groups such as $\mathrm{OH}$, $\mathrm{N}-\mathrm{O}, \mathrm{C}=\mathrm{C}, \mathrm{C}-\mathrm{N}$, and $\mathrm{C}=\mathrm{CH}_{2}$ may have an effective role in the synthesis of AgNPs. The probable chemical reactions showed that the synthesis and stabilization of AgNPs are mainly achieved by the phenols, ether, sterols, and fatty acids present in the extract. Higher content of lipid, fatty acid, and glycosaminoglycan (GAG) has been reported from the polychaete species, Sabella spallanzanii $[14,15]$.

The synthesized nanoparticle showed better antibacterial activity against all the five human pathogens evident by zone of inhibition ranging from 8 to $13 \mathrm{~mm}$ diameter. The maximum antibacterial activity was observed against Staphylococcus aureus and least antibacterial activity was observed against Vibrio parahaemolyticus. Previous study on antibacterial activity of the crude methanolic extract from polychaete has shown maximum inhibition $(8 \mathrm{~mm})$ against $S$. aureus [13]. Though silver nanoparticles find use in many antibacterial applications, the mechanism of action on microbes is still obscure. However, there are various proposed mechanisms involved in cell lysis and growth inhibition which shows that the inhibition is due to ionic binding of the AgNPs on the surface of the bacteria which creates a great intensity of the proton motive force [8]. Moreover, the small size of these particles facilitates the penetration through cell membranes and affects intracellular processes [10]. Silver nanoparticles have been reported to have strong bactericidal activity against Gram-negative, Gram-positive and multidrug resistant bacterial strains [28].

\section{Conclusion}

The present study reports the synthesis of silver nanoparticle from marine polychaete by an economical, efficient, ecofriendly, and simple process. The characterization study by UV-Vis spectroscopy, AFM, SEM, EDX, XRD, and FTIR analysis evidenced the formation of AgNPs. The synthesized nanoparticles showed promising antimicrobial activity against human pathogenic bacterial strains. The silver nanoparticle solution exhibited excellent stability for six months from the date of synthesis. The possible mechanism responsible for the synthesis of AgNPs was also illustrated. To the best of our knowledge, this is the first report on synthesis of silver nanoparticles from marine polychaete. This study provides the baseline information for synthesis of colloidal particle from marine resources especially polychaetes and calls for further exploration of the unexplored marine flora and fauna for their potent novel applications.

\section{Conflict of Interests}

The authors declare that there is no conflict of interests regarding the publication of this paper.

\section{Acknowledgments}

The authors are thankful to the authorities of Annamalai University for providing necessary facilities to carry out this work. Reena Singh is thankful to University Grant Commission, Center with Potential for Excellence in Particular Area (CPEPA), Government of India, New Delhi, for providing financial support. Sunil Kumar Sahu acknowledges the Department of Science and Technology, Government of India, New Delhi, for providing INSPIRE fellowship.

\section{References}

[1] D. Inbakandan, C. Kumar, L. S. Abraham, R. Kirubagaran, R. Venkatesan, and S. A. Khan, "Silver nanoparticles with anti microfouling effect: a study against marine biofilm forming bacteria," Colloids and Surfaces B: Biointerfaces, vol. 111, pp. 636643, 2013.

[2] R. Das, M. Saha, S. A. Hussain, and S. S. Nath, "Silver nanoparticles and their antimicrobial activity on a few bacteria," BioNanoScience, vol. 3, no. 1, pp. 67-72, 2013. 
[3] G. J. H. Lara, F. R. Gomez, G. P. Tamez, C. E. Monreal, G. R. Tamez, and P. C. Rodriguez, "In vivo antitumor activity of metal silver and silver nanoparticles in the L5178Y-R murine lymphoma model," British Journal of Medicine and Medical Research, vol. 3, no. 4, pp. 1308-1316, 2013.

[4] P. Pourali, M. Baserisalehi, S. Afsharnezhad et al., "The effect of temperature on antibacterial activity of biosynthesized silver nanoparticles," BioMetals, vol. 26, no. 1, pp. 189-196, 2013.

[5] K. Kim, W. S. Sung, B. K. Suh et al., "Antifungal activity and mode of action of silver nano-particles on Candida albicans," BioMetals, vol. 22, no. 2, pp. 235-242, 2009.

[6] H. H. Lara, N. V. Ayala-Nuñez, L. Ixtepan-Turrent, and C. Rodriguez-Padilla, "Mode of antiviral action of silver nanoparticles against HIV-1," Journal of Nanobiotechnology, vol. 8, article 1, 2010.

[7] Z. Lu, K. Rong, J. Li, H. Yang, and R. Chen, "Size-dependent antibacterial activities of silver nanoparticles against oral anaerobic pathogenic bacteria," Journal of Materials Science: Materials in Medicine, vol. 24, no. 6, pp. 1465-1471, 2013.

[8] V. K. Sharma, R. A. Yngard, and Y. Lin, "Silver nanoparticles: green synthesis and their antimicrobial activities," Advances in Colloid and Interface Science, vol. 145, no. 1-2, pp. 83-96, 2009.

[9] R. Sankar, A. Karthik, A. Prabu, S. Karthik, K. S. Shivashangari, and V. Ravikumar, "Origanum vulgare mediated biosynthesis of silver nanoparticles for its antibacterial and anticancer activity," Colloids and Surfaces B: Biointerfaces, vol. 108, pp. 80-84, 2013.

[10] A. Ravindran, P. Chandran, and S. S. Khan, "Biofunctionalized silver nanoparticles: advances and prospects," Colloids and Surfaces B Biointerfaces, vol. 105, pp. 342-352, 2013.

[11] B. V. Bhimba, J. Meenupriya, E. L. Joel, D. E. Naveena, S. Kumar, and M. Thangaraj, "Antibacterial activity and characterization of secondary metabolites isolated from mangrove plant Avicennia officinalis," Asian Pacific Journal of Tropical Medicine, vol. 3, no. 7, pp. 544-546, 2010.

[12] N. Asmathunisha and K. Kathiresan, "A review on biosynthesis of nanoparticles by marine organisms," Colloids and Surfaces B Biointerfaces, vol. 103, pp. 283-287, 2013.

[13] S. Elayaraja, P. Murugesan, S. Vijayalakshmi, and T. Balasubramanian, "Antibacterial and antifungal activities of polychaete Perinereis cultrifera," Indian Journal of Marine Sciences, vol. 39, no. 2, pp. 257-261, 2010.

[14] R. Singh, S. K. Sahu, and M. Thangaraj, "Polychaete fatty acids as potential inhibitor against human glioblastoma multiforme," International Journal of Recent Scientific Research, vol. 4, no. 10, pp. 1519-1524, 2013.

[15] L. Stabili, B. Sicuro, F. Daprà et al., "The Biochemistry of Sabella spallanzanii (Annelida: Polychaeta): a potential resource for the fish feed industry," Journal of the World Aquaculture Society, vol. 44, no. 3, pp. 384-395, 2013.

[16] P. J. W. Olive, S. W. Rees, and A. Djunaedi, "Influence of photoperiod and temperature on oocyte growth in the semelparous polychaete Nereis (Neanthes) virens," Marine Ecology Progress Series, vol. 172, pp. 169-183, 1998.

[17] A. W. Bauer, W. M. Kirby, J. C. Sherris, and M. Turck, "Antibiotic susceptibility testing by a standardized single disk method," American Journal of Clinical Pathology, vol. 45, no. 4, pp. 493496, 1966.

[18] K. Kathiresan, S. Manivannan, M. A. Nabeel, and B. Dhivya, "Studies on silver nanoparticles synthesized by a marine fungus, Penicillium fellutanum isolated from coastal mangrove sediment," Colloids and Surfaces B Biointerfaces, vol. 71, no. 1, pp. 133-137, 2009.
[19] K. Govindaraju, V. Kiruthiga, S. Tamilselvan, and G. Singaravelu, "Biogenic silver nanoparticles by Solanum torvum and their promising antimicrobial activity," Journal of Biopesticides, vol. 3, no. 1, pp. 394-399, 2010.

[20] N. Roy and A. Barik, "Green synthesis of silver nanoparticles from the unexploited weed resources," International Journal of Nanotechnology, vol. 4, pp. 95-101, 2010.

[21] A. L. Stepanov, J. R. Krenn, H. Ditlbacher et al., "Quantitative analysis of surface plasmon interaction with silver nanoparticles," Optics Letters, vol. 30, no. 12, pp. 1524-1526, 2005.

[22] G. Wang, C. Shi, N. Zhao, and X. Du, "Synthesis and characterization of Ag nanoparticles assembled in ordered array pores of porous anodic alumina by chemical deposition," Materials Letters, vol. 61, pp. 3795-3797, 2007.

[23] D. Mandal, M. E. Bolander, D. Mukhopadhyay, G. Sarkar, and P. Mukherjee, "The use of microorganisms for the formation of metal nanoparticles and their application," Applied Microbiology and Biotechnology, vol. 69, no. 5, pp. 485-492, 2006.

[24] S. S. Shankar, A. Rai, A. Ahmad, and M. Sastry, "Rapid synthesis of $\mathrm{Au}, \mathrm{Ag}$, and bimetallic Au core-Ag shell nanoparticles using Neem (Azadirachta indica) leaf broth," Journal of Colloid and Interface Science, vol. 275, no. 2, pp. 496-502, 2004.

[25] A. Sileikaite, I. Prosycevas, J. Puiso, A. Juraitis, and A. Guobiene, "Analysis of silver nanoparticles produced by chemical reduction of silver salt solution," Materials Science, vol. 12, no. 4, 2006.

[26] M. Vijayakumar, K. Priya, F. T. Nancy, A. Noorlidah, and A. B. A. Ahmed, "Biosynthesis, characterisation and anti-bacterial effect of plant-mediated silver nanoparticles using Artemisia nilagirica," Industrial Crops and Products, vol. 41, no. 1, pp. 235240, 2013.

[27] A. Nabikhan, K. Kandasamy, A. Raj, and N. M. Alikunhi, "Synthesis of antimicrobial silver nanoparticles by callus and leaf extracts from saltmarsh plant, Sesuvium portulacastrum L.", Colloids and Surfaces B: Biointerfaces, vol. 79, no. 2, pp. 488-493, 2010.

[28] M. K. Rai, S. D. Deshmukh, A. P. Ingle, and A. K. Gade, "Silver nanoparticles: the powerful nanoweapon against multidrugresistant bacteria," Journal of Applied Microbiology, vol. 112, no. 5, pp. 841-852, 2012. 

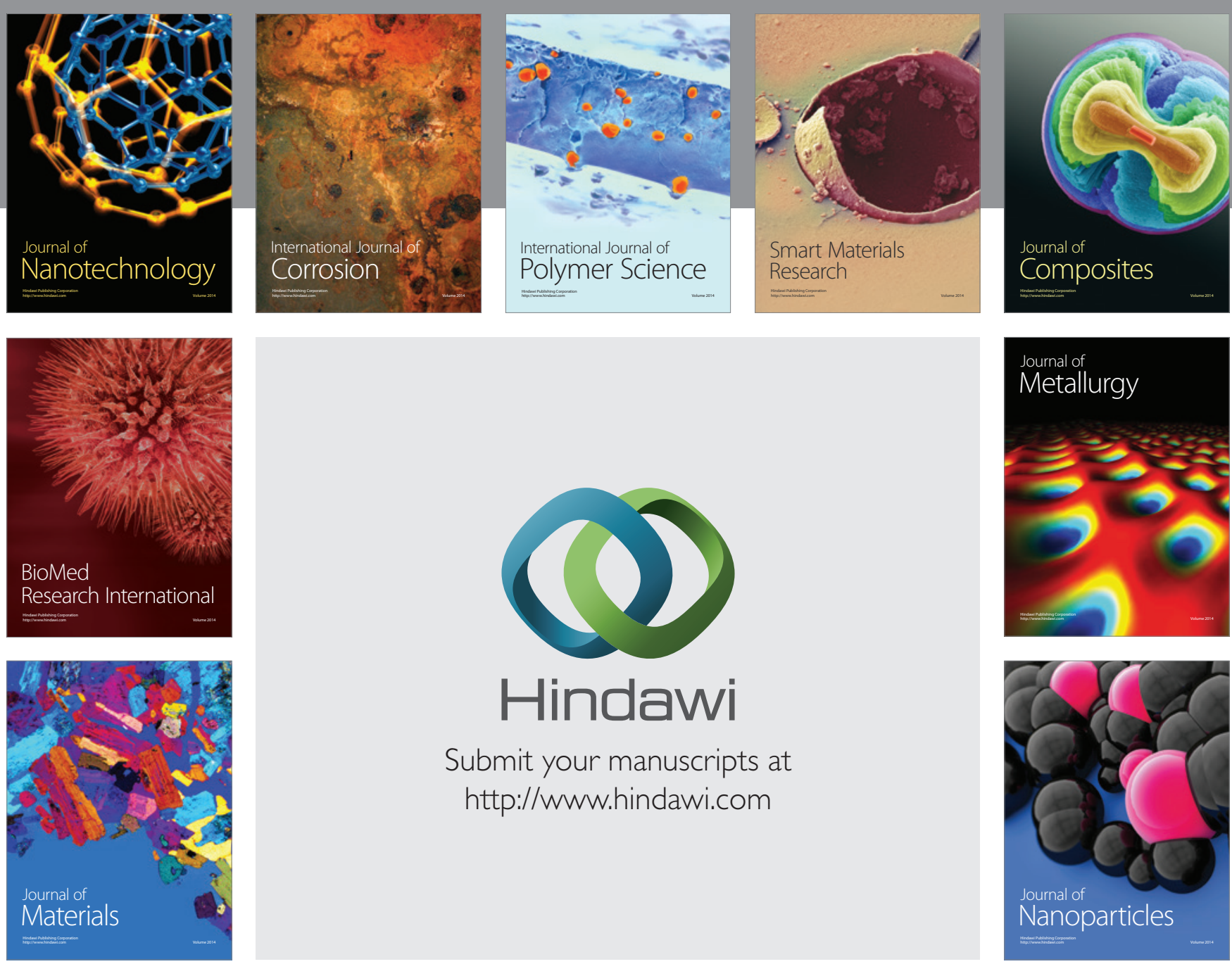

Submit your manuscripts at http://www.hindawi.com
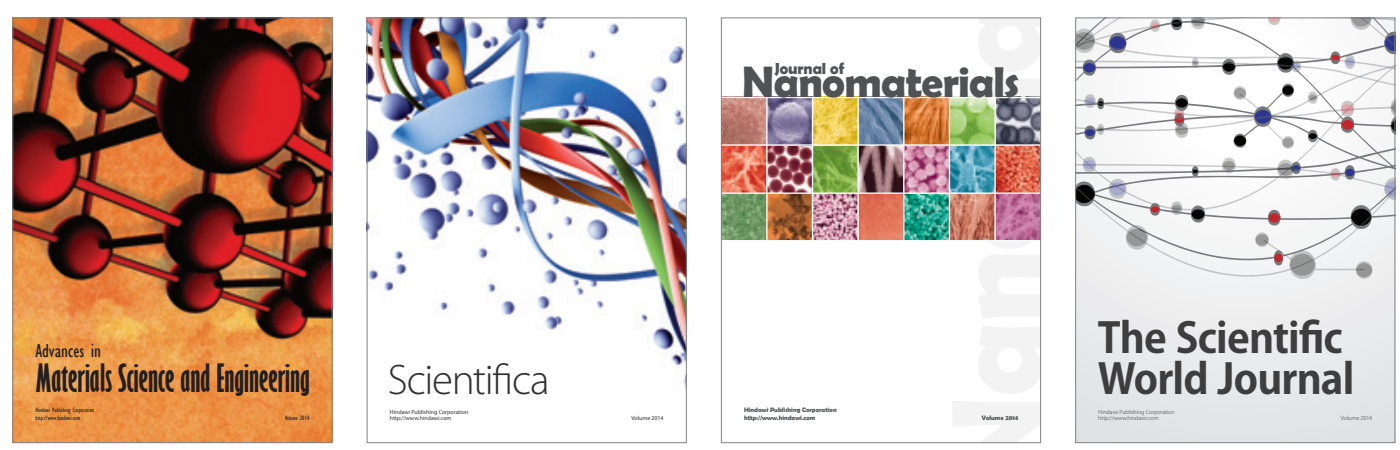

\section{The Scientific World Journal}
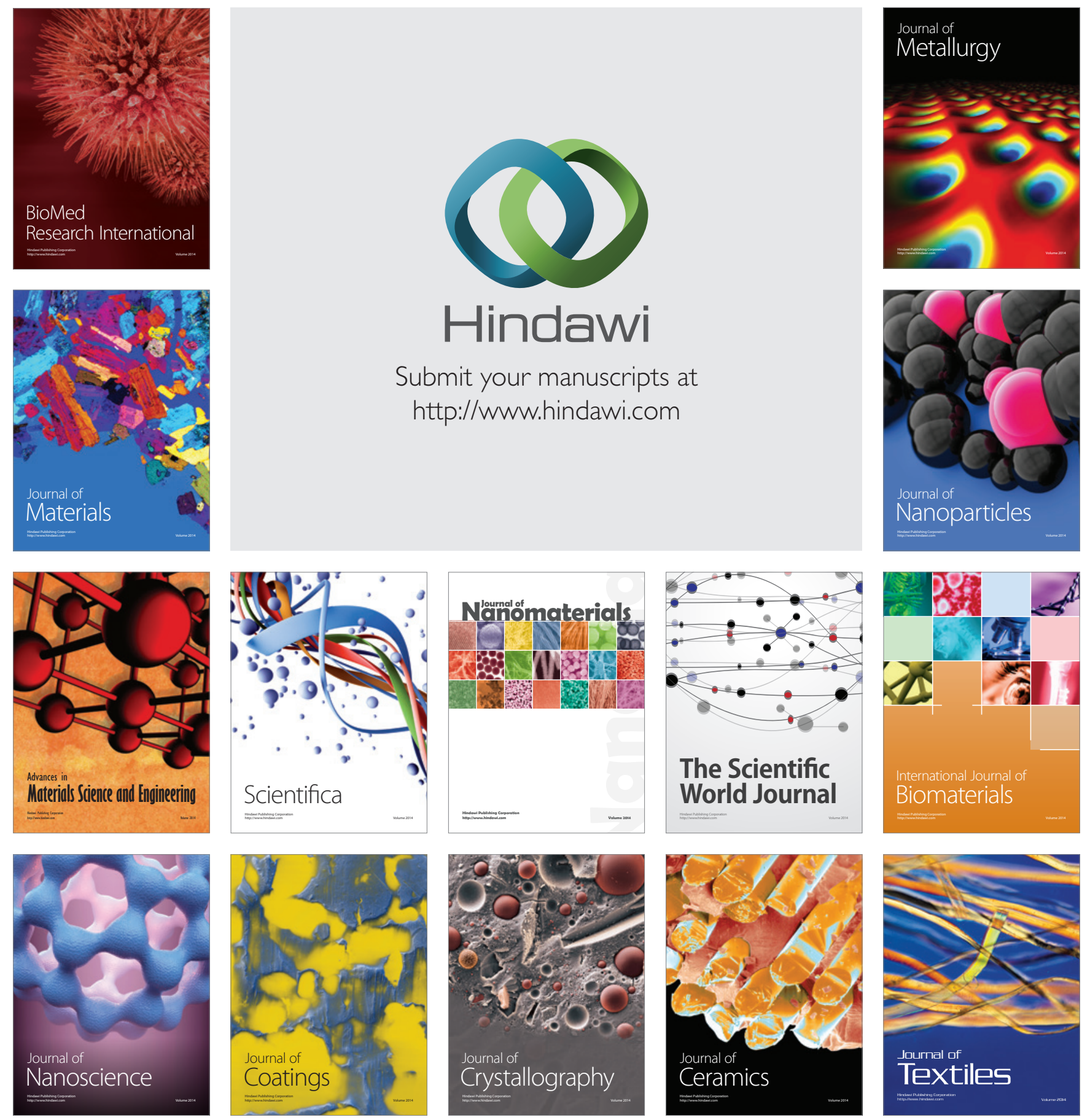